\title{
Immunoglobulin-G Enzyme-Linked Immunosorbent Assay Predicts Neutralising Antibody Response in Convalescent SARS-CoV-2 Patients
}

\section{Grant A Kay}

Liverpool School of Tropical Medicine

\section{Sophie I Owen}

Liverpool School of Tropical Medicine

\section{Emanuele Giorgi}

Lancaster University Medical School, Lancaster University

\section{Christopher T Williams}

Liverpool School of Tropical Medicine

\section{Stefanie Menzies}

Liverpool School of Tropical Medicine

\section{David J Clark}

St George's University of London

\section{Luis Cuevas}

Liverpool School of Tropical Medicine

\section{Benedict M O Davies}

St George's University of London

Nicholas M Eckersley

St George's University of London

\section{Grant L Hughes}

Liverpool School of Tropical Medicine

\section{Daniela E Kirwan}

St George's University of London

\section{Sanjeev Krishna}

St George's University of London

\section{Edward I Patterson}

Brock University

\section{Tim Planche}

St George's University of London

\section{Henry M Staines}

St George's University of London

Emily R Adams ( $\sim$ Emily.Adams@lstmed.ac.uk) 


\section{Research Article}

Keywords: SARS-COV-2, neutralising antibodies, PRNTs, ELISA

Posted Date: April 12th, 2021

DOI: https://doi.org/10.21203/rs.3.rs-329341/v1

License: (c) (1) This work is licensed under a Creative Commons Attribution 4.0 International License. Read Full License 
Immunoglobulin-G enzyme-linked immunosorbent assay predicts neutralising antibody response in convalescent SARS-CoV-2 patients

Grant A Kay ${ }^{1,2}$, MBChB, Sophie I Owen ${ }^{1}$, MSc, Emanuele Giorgi ${ }^{3}$, PhD, Christopher T Williams ${ }^{1}, \mathrm{MSc}$, Stefanie Menzies ${ }^{1}, \mathrm{PhD}$, David J Clark ${ }^{4}$, PhD, Luis Cuevas ${ }^{1}, \mathrm{PhD}$, Benedict M O Davies ${ }^{4}, \mathrm{MSc}$, Nicholas M Eckersley4, MSc, Grant L Hughes², PhD, Daniela E Kirwan ${ }^{4}$ MD, Sanjeev Krishna ${ }^{4,5,6,7}$, ScD, Edward I Patterson ${ }^{2,8}, \mathrm{PhD}$, Tim Planche ${ }^{4,5}, \mathrm{MD}$, Henry M Staines ${ }^{4}$, DPhil, *Emily R Adams ${ }^{1}$, PhD

\section{${ }^{1}$ Centre for Drugs and Diagnostics, Liverpool School of Tropical Medicine, Liverpool, UK}

${ }^{2}$ Departments of Vector Biology and Tropical Disease Biology, Centre for Neglected Tropical Diseases, Liverpool School of Tropical Medicine, Liverpool, UK

${ }^{3}$ Centre for Health Informatics Computing and Statistics, Lancaster University Medical School, Lancaster University, Lancaster, UK

${ }^{4}$ Centre for Diagnostics \& Antimicrobial Resistance, Clinical Academic Group in Institute for Infection \& Immunity, St George's University of London, London, UK

${ }^{5}$ St George's University Hospitals NHS Foundation Trust, UK

${ }^{6}$ Institut für Tropenmedizin, Universitätsklinikum Tübingen, Tübingen, Germany

${ }^{7}$ Centre de Recherches Médicales de Lambaréné, Gabon

${ }^{8}$ Department of Biological Sciences, Brock University, St. Catharines, Canada

Running title: Immunoglobulin-G enzyme-linked immunosorbent assay predicts neutralising antibody response in convalescent SARS-CoV-2 patients

Sources of funding: This work is supported by grants from DFID/Wellcome Trust Epidemic Preparedness coronavirus grant (220764/Z/20/Z) to ERA and LEC and the Rosetrees Trust and the John Black Charitable Foundation (grant number M959) to HMS, TP, SK, DJC, JRAF. HMS is supported by the Wellcome Trust Institutional Strategic Support Fund (204809/Z/16/Z) awarded to St. George's 
University of London. This work is also supported by the NIHR Health Protection Research Unit

(HPRU) in Emerging and Zoonotic Infections at University of Liverpool in partnership with Public Health England (PHE), the Liverpool School of Tropical Medicine and the University of Oxford (award 200907).

*Corresponding author: Emily R Adams, Liverpool School of Tropical Medicine, Liverpool, UK, E-mail: Emily.Adams@Istmed.ac.uk

Keywords: SARS-COV-2, neutralising antibodies, PRNTs, ELISA

Word count: Abstract, 197; Manuscript, 1498

\section{Abstract}

Severe acute respiratory coronavirus 2 (SARS-CoV-2) has spread globally since its emergence in 2019. Most SARS-CoV-2 infections generate immune responses leading to rising levels of immunoglobulins (Ig) M, A and $G$ which can be detected using diagnostic tests including enzymelinked immunosorbent assays (ELISA). Whilst implying previous SARS-CoV-2 infection, the detection of Ig by ELISA does not guarantee the presence of neutralising antibodies (NAb) that can prevent the virus infecting cells. Plaque reduction neutralisation tests (PRNT) detect NAb but are not amenable to mass testing as they take several days and require use of viable SARS-CoV-2 in high biocontainment laboratories. We evaluated the ability of IgG and IgM ELISAs targeting SARS-CoV-2 spike subunit 1 (S1) and nucleocapsid protein (NP) at predicting the presence and magnitude of NAb determined by PRNT. SARS-CoV-2 IgG ELISA correlated well with NAb and was highly sensitive (93.8\% [95\% Cl 79.2-99.2]) and specific (88.9\% [95\% Cl 51.8-99.7\%]) at predicting the presence of NAb. There was not a strong correlation between IgM ELISA and PRNT result. IgG ELISA provides a useful, high throughput method of predicting the presence of neutralising antibodies, with higher ELISA results increasing the likelihood of having a greater NAb titre.

\section{Introduction}

Severe acute respiratory syndrome coronavirus 2 (SARS-CoV-2) is a novel, pandemic betacoronavirus that began spreading globally in early 2020. To date, there have been over 111 million reported infections and more than 2.4 million deaths [1]. 
Most individuals infected with SARS-CoV-2 develop humoral immune responses, characterised by rising titres of immunoglobulins (Ig) M, A and $G$, within the first 2-3 weeks of infection [2, 3], which are detectable using enzyme-linked immunosorbent assays (ELISA). The presence of SARS-CoV-2 specific Ig therefore provides evidence of previous infection [4], although their detection does not guarantee the presence of functional immunity against the virus [5]. For example, the viral nucleocapsid protein (NP), an abundant viral antigen, generates robust antibody responses, and is therefore a good antigen for diagnostic serological assays [6], however these antibodies are not neutralising $[7,8]$.

Neutralising antibodies (NAb) primarily bind the receptor-binding domain of the spike (S) protein and disrupt virus entry by blocking interaction with the angiotensin converting enzyme 2 (ACE2) receptor of host cells $[7,9]$. The activity of these functional antibodies can be measured using the plaque reduction neutralisation test (PRNT). However, this method is not amenable to mass testing, as the process takes several days and requires working with SARS-CoV-2 in high biocontainment laboratories, or with less pathogenic pseudotyped virus models. Previous studies have reported that NAb levels correlate with IgG and IgM titres [10-13], but this relationship is variable, depending on the timing of sampling in the course of the infection and the antigen targets of the serological assays [14]. Here we evaluate the ability of SARS-CoV-2 IgG and IgM ELISAs to predict the presence and magnitude of SARS-CoV-2 NAbs in convalescent COVID-19 patients.

\section{Methods}

\section{Ethical statement}

72

The study was conducted in accordance with relevant UK guidelines and regulations. Ethics approval was provided by the Institutional Review Board (South Central - Oxford C Research Ethics Committee, Research Development and Assessment of Rapid Testing for SARS-CoV-2 outbreak study; Integrated Research Application System project ID:282104; Research Ethics Committee Reference 20/SC/0171; registered at clini-caltrials.gov NTC04351646). The approved protocol permitted the analysis of antibody responses using anonymised excess diagnostic material (EDM) from the pathology laboratory of patients with and without PCR-confirmed SARS-CoV-2 infection. Informed consent was not required under the ethical approval status of the work and due to the nature of the samples. 
84

Anonymized EDM serum samples from hospital patients with SARS-CoV-2 infection confirmed by reverse transcription - quantitative polymerase chain reaction (RT-qPCR) were used for this study and were selected from 645 EDM serum samples that were collected from a pool of 177 patients treated at St George's Hospital, London UK [15]. Where possible, samples were selected from patients at least 10 days post-RT-qPCR confirmation. Samples were grouped based on their normalised optical density (NOD) values derived from an anti-SARS-CoV-2 IgG ELISA [15] into "negative NOD" values (<0; indicating the patient had not seroconverted), "low NOD" (0 to 0.5), "medium NOD" (0.9 to 1.1); and "high NOD" (> 1.5). The final sample available from all patients was chosen for this study. The narrow "medium NOD" window was purposely selected to reduce sample numbers in this grouping, as the grouping 0.5 to 1.5 contained 5-6 times more samples than the other groupings. A single sample was then selected from any patient with at least 3 samples with NOD values remaining in one NOD grouping (i.e. indicating a stable antibody response). The serum sample selected for any given patient was that collected furthest from the swab taken for confirmation of SARS-CoV-2 infection (and at least 10 days post-swab). The approach resulted in 9, 9, 11 and 12 patient samples in each group (41 single patient serum samples in total).

All participants were confirmed as positive for SARS-CoV-2 using RT-PCR from nose/throat swabs (in Sigma Virocult ${ }^{\circledR}$, Corsham, UK) and Roche RNA extraction kits (Magnapure, West Sussex, UK) followed by Altona Diagnostics RealStar ${ }^{\circledR}$ SARS-CoV-2 RT-PCR (S and E target genes, Hamburg, Germany) or Roche cobas ${ }^{\circledR}$ SARS-CoV-2 Test (E and ORF target genes).

Enzyme-linked immunosorbent assays to detect anti-SARS-CoV-2 IgM

Anti-SARS-CoV-2 IgM ELISAs (Mologic, Bedfordshire, UK), which targets the nucleocapsid (NP) and spike protein subunit 2 (S2) antigens, were used to measure antibodies, as per the manufacturer's instructions. Briefly, sera were diluted and incubated on a pre-coated plate (30 minutes) at room temperature and then washed three times. Conjugated antibody (anti-human IgM) was then applied to each well and incubated (30 minutes) at room temperature. Following washing $(x 4), T M B$ substrate was added and incubated for 10 minutes at room temperature before addition of stop solution. Optical densities (OD) were read at $450 \mathrm{~nm}$ within 10 minutes of addition of the stop solution.

\section{Plaque reduction neutralisation tests}

Vero E6 cells were seeded into 24 -well cell culture plates at a density of 250,000 cells/ml and incubated $\left(24\right.$ hours, $37^{\circ} \mathrm{C}, 5 \% \mathrm{CO}_{2}$ ). The following day serum samples were heated to inactivate 
complement $\left(56^{\circ} \mathrm{C}\right.$ for 1 hour). Heat-inactivated serum samples were 2 -fold serially diluted in infection media (DMEM with 2\% v/v FBS and 1:1000 50mg/ml gentamicin). Under biosafety level 3 conditions, SARS-CoV-2 isolate REMRQ0001/Human/2020/Liverpool [16] was added to an equal volume of diluted patient serum, at a titre of $800 \mathrm{pfu} / \mathrm{ml}$, to achieve 12 final serum dilutions from 1:20 to 1:40960 for each patient sample. Following incubation ( 1 hour, $\left.37^{\circ} \mathrm{C}\right)$, the virus-serum mixture $(100 \mu \mathrm{L})$ was inoculated onto Vero $\mathrm{E} 6$ cells and incubated $\left(1 \mathrm{hour}, 37^{\circ} \mathrm{C}, 5 \% \mathrm{CO}_{2}\right.$ ) before applying an overlay of infection media containing agarose $(0.4 \% \mathrm{w} / \mathrm{v})$. Infected cells were then incubated ( 48 hours, $\left.37^{\circ} \mathrm{C}, 5 \% \mathrm{CO}_{2}\right)$. The assays were fixed with formaldehyde $(37 \% \mathrm{w} / \mathrm{v})$, stained with crystal violet solution $(0.25 \% \mathrm{w} / \mathrm{v})$ and allowed to air dry. The PRNT 80 was determined as the lowest dilution of serum that produced $a \geq 80 \%$ reduction in the number of plaques compared to controls that contained no patient serum. The investigators were blinded to the ELISA status of the samples when performing the PRNTs.

Western blots

Western blots were conducted to investigate the antigen binding profiles associated with the neutralising responses. Recombinant spike subunit 1 protein (S1), spike subunit 2 protein (S2) and nucleoprotein (NP) (Native Antigen Company, Kidlington, UK) were prepared in NuPAGE LDS sample buffer (Invitrogen, Carlsbad, USA) with 50 mM dithiothreitol (Sigma, St Louis, USA), and heated for 10 minutes at $70^{\circ} \mathrm{C}$. Protein $(1 \mu \mathrm{g})$ was loaded on a Mini-PROTEAN TGX $12 \%$ PAGE gel (BioRad, Hercules, USA) and run under reducing conditions in Tris/Glycine/SDS Buffer (BioRad, Hercules, USA). Proteins were transferred to $0.2 \mu \mathrm{m}$ nitrocellulose membrane using BioRad TransBlot Turbo system mixed MW programme, and blocked in 5\% (v/v) goat serum (Sigma, St Louis, USA) in PBS (Gibco, Waltham, USA) with $0.1 \%(\mathrm{v} / \mathrm{v})$ Tween 20 (Sigma, St Louis, USA) overnight at $4^{\circ} \mathrm{C}$ with gentle shaking. Blots were incubated with sera diluted 1 in 200 in blocking solution for 1 hour at room temperature with gentle shaking and washed three times for five minutes with PBS-0.1\% (v/v) Tween 20. Blots were then incubated with goat anti-human Kappa-AP and anti-human Lambda-AP (Southern Biotech, Birmingham, USA), both diluted 1 in 1000 in blocking solution, for 1 hour at room temperature with gentle shaking, followed by three washes as above. Blots were developed with BCIP/NBT (Sigma, St Louis, USA) for 1 minute and stopped with $\mathrm{H}_{2} \mathrm{O}$. The investigators were blinded to the ELISA status of the samples. One IgG ELISA negative sample was excluded because there was not enough serum remaining for western blot. The investigators were blinded to the ELISA status of the samples when performing the western blots. 
A statistical model for ordinal outcomes was developed and fitted to estimate the association between IgG, IgM and timing of serum sampling post-symptom onset with PRNT 80 . Briefly, the PRNT 80 outcome for the $\mathrm{i}$-th patient, denoted by $Y_{i}$, was modelled as an ordinal variable taking the values from $\mathrm{k}=1$, corresponding to a titre less than 1:20, to $k=10$, for a titre of 1:2560 or more. We assumed that the observed titre values corresponded to the discretization of a continuous antibody distribution, $Y_{i}^{*}$, which was assumed to follow a log-Gaussian distribution with mean $\vartheta_{i}$ and variance $\sigma^{2}$.

This can be summarised as:

$$
P\left(Y_{i}=k\right)=P\left(a_{k}<Y_{i}^{*}<a_{k+1}\right), k=1, \ldots, 10,
$$

where $a_{1}=0, a_{2}=20, a_{3}=40$ and so forth up $a_{10}=2560$ and with the convention $a_{11}=\infty$.

The probability in (1) is computed using the cumulative density function of a log-Gaussian distribution.

We model the mean concentration $\vartheta_{i}$ of PRNT 80 as a log-linear regression on $\operatorname{Ig} G_{i}$ and $\operatorname{Ig} M_{i}$, and the days post-symptom, $d_{i}$, i.e.

$$
\log \left\{\vartheta_{i}\right\}=\alpha+\beta_{I g G} \operatorname{Ig} G_{i}+\beta_{I g M} \operatorname{Ig} M_{i}+\beta_{d} d_{i}
$$

where the $\alpha$ is the intercept; $\beta_{I g G}$ and $\beta_{I g M}$ express the strength of the effect of the $\operatorname{Ig} G_{i}$ and $\operatorname{Ig} M_{i}$ concentrations on the log-mean levels of PRNT 80 ; similarly, $\beta_{d}$ indicates the effect of the days post-symptoms. The model was fitted via maximum likelihood using R software (Supplementary 1).

\section{Results}

Twenty-five (61.0\%) of the 41 patients were male, and the median age was 63 (IQR 55-71) years. Seventeen patients (41.5\%) were classified as white, 13 (31.7\%) non-white, and 11 (26.8\%) were of unknown or 'other' ethnicity. Twenty-three patients $(56.1 \%)$ had one or more comorbidities. Ten patients (24.4\%) were obese, with a body mass index $(\mathrm{BMI})>30$. These patients come from a subset already described [15].

Of the thirty-nine patients for whom symptom data was available, $35(89.7 \%)$ were symptomatic at the time of their initial swab. Thirty-three of these patients $(94.3 \%)$ had one or more of the classic triad of symptoms: cough, fever, and shortness of breath, 12 (34.3\%) had gastrointestinal symptoms, including 9 patients with diarrhoea. Three patients (7.3\%) had an incidental positive swab, taken 
prior to admission to a rehabilitation facility, and three patients were swabbed following contact with a patient who had tested positive. The median interval between the onset of symptoms and date of the first positive swab was 4 days (IQR 3-7 days). Seven (17\%) patients died within 28 days of their first positive swab. The median timing of serum sampling post symptom onset was 29 days (range 13-60 days). The sample timing post-symptom onset was not available for six patients.

The correlation of IgG ELISA and neutralising responses are shown in Figure 1. Using an $\mathrm{NOD}_{450}$ of 0.2 , the IgG ELISA was $93.8 \%$ [95\% Cl 79.2-99.2] sensitive, and 88.9\% [95\% Cl 51.8-99.7] specific at predicting a PRNT $80 \geq 1: 40$ (Table 1). For IgM, an $\mathrm{OD}_{450}$ cut-off of 0.3 was $75.0 \%$ [95\% $\mathrm{Cl}$ 56.6-88.5] sensitive, and $75.0 \%$ [95\% $\mathrm{Cl} 34.9-96.8]$ specific at predicting a $\mathrm{PRNT}_{80} \geq 1: 40$. There was insufficient volume of one sample to perform the IgM ELISA.

One sample was positive by IgG ELISA (NOD ${ }_{450} 0.41$ ) but did not show a neutralising response $\left(\mathrm{PRNT}_{80}<1: 20\right)$. An IgG negative ( $\left.\mathrm{NOD}_{450}-0.1\right)$ sample and one sample with borderline negative IgG $\left(N_{450}\right.$ 0.07) demonstrated a neutralising titre of $\mathrm{PRNT}_{80}$ 1:40 (Figure 1B). There was considerable variation in the relative titres of IgG and IgM for each patient, with the majority of patients who demonstrated a high $\operatorname{PRNT}_{80}(\geq 1: 320$ ) having relatively higher IgG than IgM titres (Figure 1C). Neutralising activity achieving PRNT 80 was seen down to a 1:2560 dilution of patient serum for both IgG and IgM.

An ordinal outcomes model was fitted to explore the effects of $\lg G, \operatorname{lgM}$, and the timing of serum sampling post-onset of symptoms on the degree of neutralisation. The maximum likelihood estimates are shown in Table 2. IgG, denoted as $\beta / g G$, was a significant predictor of PRNT 80 , with each unit increase in IgG titre associated with a 0.626 [95\% Cl 0.291-0.961] increased likelihood of being in a higher PRNT 80 category. Neither $\operatorname{IgM}(\beta / g M)$, nor time of serum sampling $(\beta d)$, had a significant relationship with the observed PRNT ${ }_{80}$. As IgG ELISA result was the only significant explanatory variable, IgM ELISA and timing of serum sampling post-symptom onset were excluded from subsequent analysis.

It was assumed that the relationship between IgG titre and PRNT ${ }_{80}$ was unlikely to be linear across all ranges of observed IgG. Therefore, the model predictions for $\mathrm{PRNT}_{80}$ based on $\lg \mathrm{G}$ were considered for the first, median, and third quartiles of the IgG titres (see Figure 2). For the first quartile, there was a low probability $(<0.2)$ of observing neutralising titres $\geq 1: 20$ (Figure $2 \boldsymbol{A}$ ). For a median IgG titre, the highest probability (0.15) was for PRNT ${ }_{80} 1: 160$ (Figure 2B). IgG ELISA results in the $3^{\text {rd }}$ quartile had a generally higher probability of a higher $\mathrm{PRNT}_{80}$ than median or $1^{\text {st }}$ quartile IgG titres, albeit with increasing uncertainty at higher $\mathrm{PRNT}_{80}$ values (Figure $\mathbf{2 C}$ ). Across the range of IgG titres 
observed, we predicted that increasing IgG corresponded to $\mathrm{PRNT}_{80}$, but with greater uncertainty in predicting the neutralising response at higher IgG titres (Figure 2D).

Antibody binding to all three of the S1, S2 and NP antigens, or to both the S2 and NP antigens, were seen in all samples that had high neutralising titres (PRNT $80 \geq 1: 80$ ) (Figure 3). In samples with low PRNT $_{80}(\leq 1: 40)$ there was greater variability in antigen binding, with a larger proportion demonstrating antibody binding to single antigens, or combinations of two antigens involving S1. One IgM and IgG positive sample by ELISA showed binding to only NP. Three of the six samples that did not achieve PRNT $80 \geq 1: 20$ demonstrated no binding to $\mathrm{S} 1$, S2 or NP.

\section{Discussion}

Our data show that IgG ELISA (NOD 450 cut-off 0.2 ) can predict the presence of a NAb titre of $\geq P R N T_{80}$ 1:40 with high sensitivity (93.8\%) and specificity (88.9\%), and therefore can be used as a proxy of neutralising response to SARS-CoV-2 in convalescent patients. Our ordinal outcomes model demonstrates higher NAb titres correlate with increasing IgG titres. These findings are supported by previous studies, which report a significant correlation between anti-spike and anti-RBD IgG titres with the neutralisation titres established by microneutralisation tests, PRNTs and pseudotyped virus neutralisation assays $[6,14,17-19]$. However, our findings also demonstrate considerable IgG NOD variation for samples within the same $\mathrm{PRNT}_{80}$ category, indicating it is not possible to make accurate quantifiable predictions of the expected $\mathrm{PRNT}_{80}$ based on the IgG ELISA titres alone.

The IgM ELISA NOD was not significantly associated with the NAb titres and was less sensitive and specific at identifying a neutralising response ( $\geq \mathrm{PRNT}_{80} 1: 40$ ) than the IgG ELISA. Previously, IgM ELISAs have been reported to be more predictive of neutralising titres than $\operatorname{IgG}[13,17,20]$. Given the short duration of IgM expression, the timing of serum sampling post-infection is an important determinant of these relationships $[13,17,20]$. Furthermore, as serum samples were selected to provide a range of IgG titres, it is possible that a larger sample size could have provided a wider range of IgM titres to detect a relationship. IgA titres correlate well with neutralisation titres [21]. As we did not measure IgA, it is possible that its neutralising activity may explain why some samples had greater PRNT 80 values than expected from the IgG titres.

Anti-NP antibodies are not considered to have protective activity, despite correlating with neutralising titres [2]. Our western blot analysis revealed that the majority of serum samples with neutralising activity had antibodies directed against the NP, S1 and S2. This finding suggests that anti-NP antibodies are raised as part of a suite of antibodies, and whilst not directly neutralising, are indicative of the presence of other neutralising immunoglobulins. In general, the inclusion of the NP 
in an ELISA does not seem to prevent accurate prediction of the presence of a neutralising response.

243 For example, the Roche Elecsys Anti-SARS-CoV-2 ELISA, which targets only the NP has a similar performance predicting neutralisation as the Abbott SARS-CoV-2 IgG ELISA, which targets both the NP and S1 [22]. However, one sample with a PRNT ${ }_{80}<1: 20$ which was positive by IgG and IgM ELISA in our study, only showed binding to the NP on the western blot. This highlights how isolated NP binding is capable of producing ELISA positive results which are not associated with neutralisation.

The main limitation of our study is the small sample size. As the relationship between serological assays and neutralising titres appears to be variable, a larger sample size would have sufficient power to reveal overall trends and minimise the effects of outliers. Moreover, our cohort only includes hospitalised patients with severe COVID-19 and therefore the findings of this study may not be applicable to individuals with mild and asymptomatic infections, who may foster different antibody responses.

In conclusion, IgG ELISA targeting SARS-CoV-2 S2 and NP can predict the presence of a NAbs to SARS-

CoV-2 in convalescent patients hospitalised with severe COVID-19.

\section{$\underline{\text { References }}$}

1. WHO. WHO Coronavirus Disease (COVID-19) Dashboard. 2021 22.02.2021 [cited 2021 22.02.21]; Available from: https://covid19.who.int/.

2. To, K.K.-W., et al., Temporal profiles of viral load in posterior oropharyngeal saliva samples and serum antibody responses during infection by SARS-CoV-2: an observational cohort study. The Lancet Infectious Diseases, 2020. 20(5): p. 565-574.

3. Long, Q.-X., et al., Antibody responses to SARS-CoV-2 in patients with COVID-19. Nature Medicine, 2020. 26(6): p. 845-848.

4. Adams, E., et al., Evaluation of antibody testing for SARS-CoV-2 using ELISA and lateral flow immunoassays. medRxiv, 2020: p. 2020.04.15.20066407.

5. VanBlargan, L.A., L. Goo, and T.C. Pierson, Deconstructing the Antiviral Neutralizing-Antibody Response: Implications for Vaccine Development and Immunity. Microbiology and Molecular Biology Reviews, 2016. 80(4): p. 989-1010.

6. Wajnberg, A., et al., SARS-CoV-2 infection induces robust, neutralizing antibody responses that are stable for at least three months. medRxiv, 2020: p. 2020.07.14.20151126.

7. Ju, B., et al., Human neutralizing antibodies elicited by SARS-CoV-2 infection. Nature, 2020. 584(7819): p. 115-119.

8. $\mathrm{Ni}$, L., et al., Detection of SARS-CoV-2-Specific Humoral and Cellular Immunity in COVID-19 Convalescent Individuals. Immunity, 2020. 52(6): p. 971-977.e3.

9. Shi, R., et al., A human neutralizing antibody targets the receptor-binding site of SARS-CoV-2. Nature, 2020. 584(7819): p. 120-124.

10. Wang, K., et al., Longitudinal Dynamics of the Neutralizing Antibody Response to Severe Acute Respiratory Syndrome Coronavirus 2 (SARS-CoV-2) Infection. Clinical Infectious Diseases, 2020.

11. Wang, Y., et al., Kinetics of viral load and antibody response in relation to COVID-19 severity. The Journal of Clinical Investigation, 2020. 130(10): p. 5235-5244.

12. Deeks, J.J., et al., Antibody tests for identification of current and past infection with SARSCoV-2. Cochrane Database Syst Rev, 2020. 6(6): p. Cd013652. 
13. Padoan, A., et al., Evaluation of an ELISA for SARS-CoV-2 antibody testing: clinical performances and correlation with plaque reduction neutralization titer. Clinical Chemistry and Laboratory Medicine (CCLM), 2020. 58(11): p. e247-e249.

14. Luchsinger, L.L., et al., Serological Assays Estimate Highly Variable SARS-CoV-2 Neutralizing Antibody Activity in Recovered COVID-19 Patients. Journal of clinical microbiology, 2020. 58(12): p. e02005-20.

15. Staines, H.M., et al., IgG Seroconversion and Pathophysiology in Severe Acute Respiratory Syndrome Coronavirus 2 Infection. Emerg Infect Dis, 2021. 27(1): p. 85-91.

16. Patterson, E.I., et al., Methods of Inactivation of SARS-CoV-2 for Downstream Biological Assays. The Journal of Infectious Diseases, 2020. 222(9): p. 1462-1467.

17. Perera, R.A., et al., Serological assays for severe acute respiratory syndrome coronavirus 2 (SARS-CoV-2), March 2020. Eurosurveillance, 2020. 25(16): p. 2000421.

18. Jungbauer, C., et al., Characterization of 100 sequential SARS-CoV-2 convalescent plasma donations. Transfusion. $\mathrm{n} / \mathrm{a}(\mathrm{n} / \mathrm{a})$.

19. Lau, E.H.Y., et al., Neutralizing antibody titres in SARS-CoV-2 infections. Nature Communications, 2021. 12(1): p. 63.

20. Padoan, A., et al., Clinical performances of an ELISA for SARS-CoV-2 antibody assay and correlation with neutralization activity. Clinica chimica acta; international journal of clinical chemistry, 2020. 510: p. 654-655.

21. Mazzini, L., et al., Comparative analyses of SARS-CoV-2 binding $(\lg G, \lg M, \lg A)$ and neutralizing antibodies from human serum samples. Journal of Immunological Methods, 2021. 489: p. 112937.

22. Tang, M.S., et al., Association between SARS-CoV-2 Neutralizing Antibodies and Commercial Serological Assays. Clinical Chemistry, 2020. 66(12): p. 1538-1547.

Authors' contributions: Conceptualization: SK, HMS, DJC, TP, ERA, LEC. Methodology: SK, HMS, DJC, TP, ERA, LEC, GLH, EIP. Investigation: GAK, SIO, CTW, SM, DJC, BMOD, NME, DEK, HMS. Formal Analysis: GAK, EG. Writing - Original Draft: GAK, SIO, CTW, SM. Writing - Review \& Editing: All.

Conflicts of interest: SK and HMS are shareholders and SK is advisor to QuantuMDx, a molecular nucleic acid test-based diagnostic company. SK is also member of the Scientific Advisory Committee for the Foundation for Innovative New Diagnostics (FIND), a not-for-profit organisation that produces global guidance on affordable diagnostics. GAK, SIO, EG, CTW, SM, DJC, LC, BMOD, NME, GLH, DEK, EIP, TP and ERA report no competing interests.

(1)


321 Table 1 - Sensitivity and specificity of IgG and IgM ELISA at a range of absorbency cut- offs

322 Table 2-Maximum likelihood estimates and 95\% confidence intervals

$323 \quad$ List of figures

324 Figure 1 - IgG NOD $450(1 \mathrm{~A})$ and IgM OD 450 (1B) by PRNT 80 category, and paired IgG and IgM ELISA 325 results for each serum sample (1C)

326

327 Fiqure 2-PRNT 80 probability estimates by IgG ELISA result

328 Figure 3 - Percentage of samples in PRNT 80 categories binding to different antigen combinations by 329 Western Blot

330

331

332

333

334

335

336

337

338

339

340

341

342

343

344

345

346

347

348

349 
Table 2-Maximum likelihood estimates and 95\% confidence intervals

\section{$\geq$ PRNT $_{80} 1: 40$}

\begin{tabular}{|c|c|c|c|}
\hline & \multicolumn{3}{|c|}{$\geq$ PRNT $_{80} 1: 40$} \\
\hline & $\begin{array}{c}\text { Absorbance } \\
\text { cut-off }\end{array}$ & $\begin{array}{c}\text { Sensitivity } \\
\text { (\%) }\end{array}$ & $\begin{array}{c}\text { Specificity } \\
\text { (\%) }\end{array}$ \\
\hline \multirow{4}{*}{ IgG } & 0.2 & $\begin{array}{c}93.8 \\
{[79.2-99.2]}\end{array}$ & $\begin{array}{c}88.9 \\
{[51.8-99.7]}\end{array}$ \\
\hline & 0.3 & $\begin{array}{c}87.5 \\
{[71.0-96.5]}\end{array}$ & $\begin{array}{c}88.9 \\
{[51.8-99.7]}\end{array}$ \\
\hline & 0.4 & $\begin{array}{c}84.4 \\
{[67.2-94.7]}\end{array}$ & $\begin{array}{c}88.9 \\
{[51.8-99.7]}\end{array}$ \\
\hline & 0.5 & $\begin{array}{c}71.9 \\
{[53.3-86.3]}\end{array}$ & $\begin{array}{c}100 \\
{[66.4-100.0]}\end{array}$ \\
\hline \multirow{4}{*}{ IgM } & 0.2 & $\begin{array}{c}100 \\
{[89.1-100.0]}\end{array}$ & $\begin{array}{c}0.0 \\
{[0.0-36.9]}\end{array}$ \\
\hline & 0.3 & $\begin{array}{c}75.0 \\
{[56.6-88.5]}\end{array}$ & $\begin{array}{c}75.0 \\
{[34.9-96.8]}\end{array}$ \\
\hline & 0.4 & $\begin{array}{c}59.4 \\
{[40.6-76.3]}\end{array}$ & $\begin{array}{c}100.0 \\
{[63.1-100.0]}\end{array}$ \\
\hline & 0.5 & $\begin{array}{c}40.6 \\
{[23.7-59.4]}\end{array}$ & $\begin{array}{c}100.0 \\
{[63.1-100.0]}\end{array}$ \\
\hline
\end{tabular}

\begin{tabular}{|c|c|c|}
\hline Parameter & Estimate & 95\% Confidence Interval \\
\hline$\alpha$ & 6.275 & $(5.197,7.353)$ \\
\hline$\beta_{I g G}$ & 0.626 & $(0.291,0.961)$ \\
\hline$\beta_{I g M}$ & 0.110 & $(-0.535,0.754)$ \\
\hline$\beta_{d}$ & -0.002 & $(-0.0370 .033)$ \\
\hline$\sigma$ & 4563.0 .85 & $(893.576,23301.600)$ \\
\hline
\end{tabular}




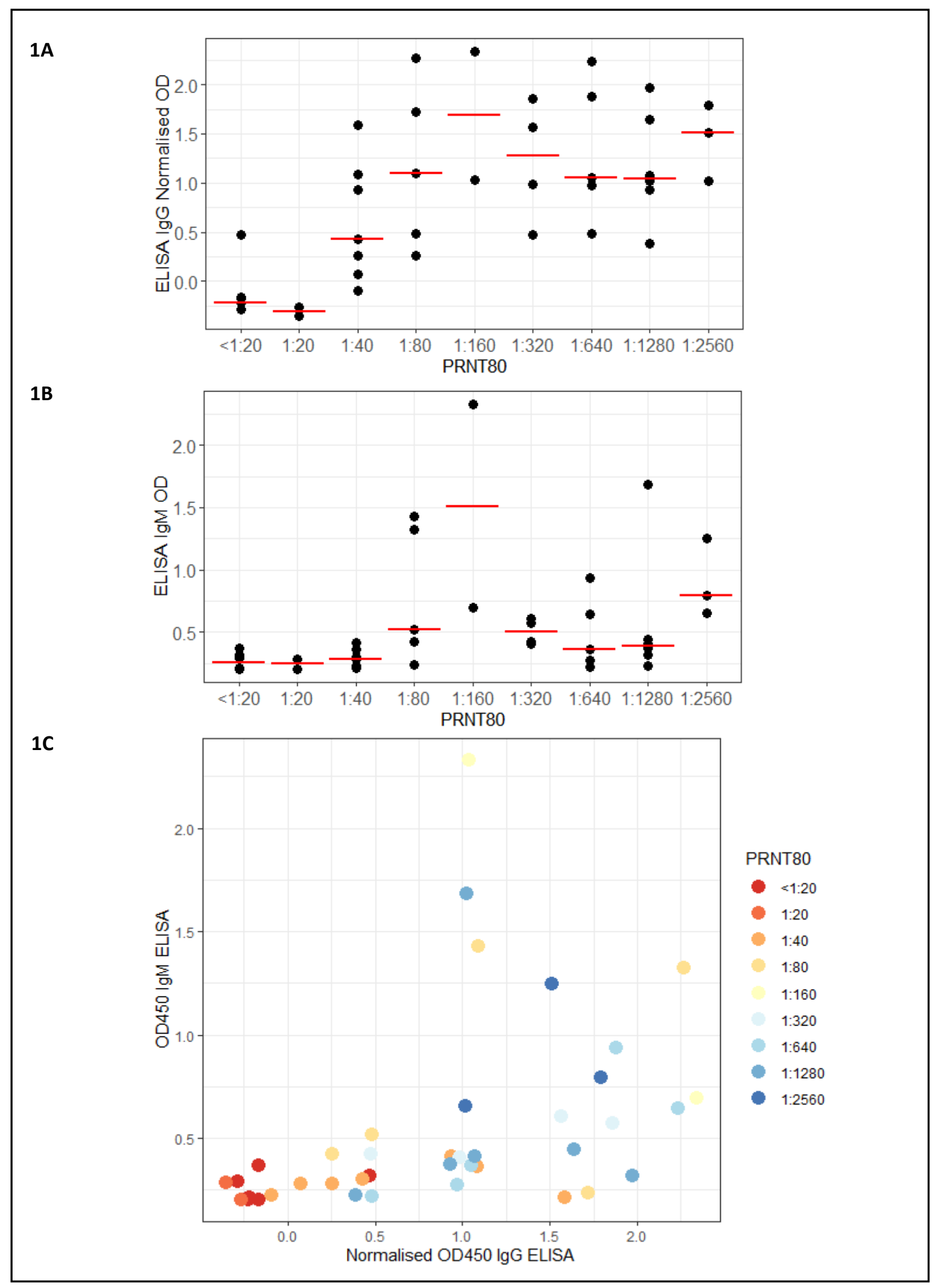

Figure 1: IgG NOD $450(1 \mathrm{~A})$ and $\operatorname{lgM} O D_{450}(1 \mathrm{~B})$ by $P R N T_{80}$ category.

Median $O D$ values are indicated by the red horizontal bars. One sample had insufficient remaining volume for IgM ELISA to be performed. Figure 1 C shows paired IgG and IgM ELISA, and PRNT 80 results for each sample 

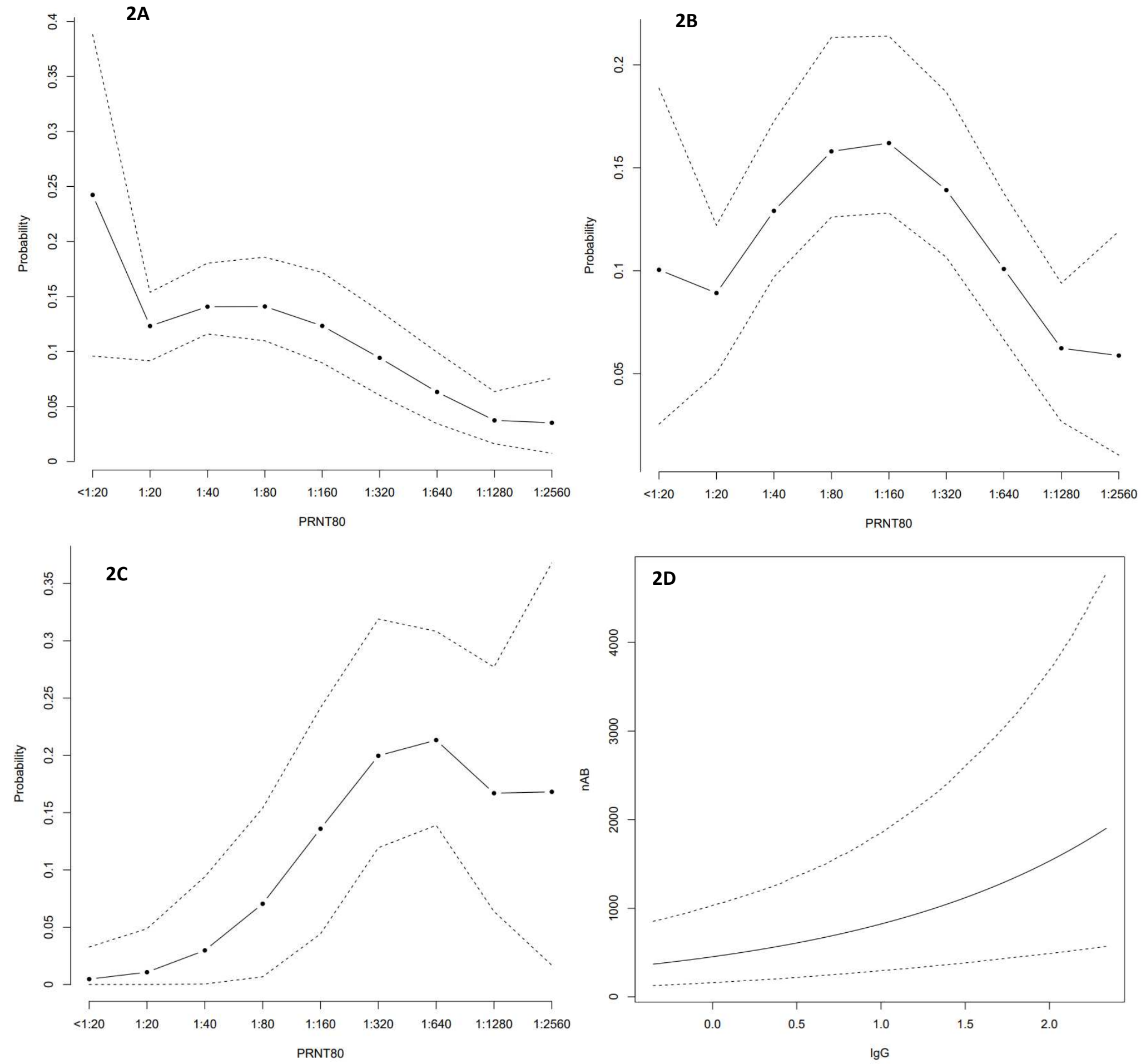

369 Figure 2: PRNT 80 probability estimates by IgG ELISA result. Figure $2 A$ : first quartile IgG ELISA results;

370 Figure 2B: median IgG ELISA results; Figure 2C: third quartile IgG ELISA results; Figure 2D: predicted

371 PRNT 80 by IgG ELISA result 


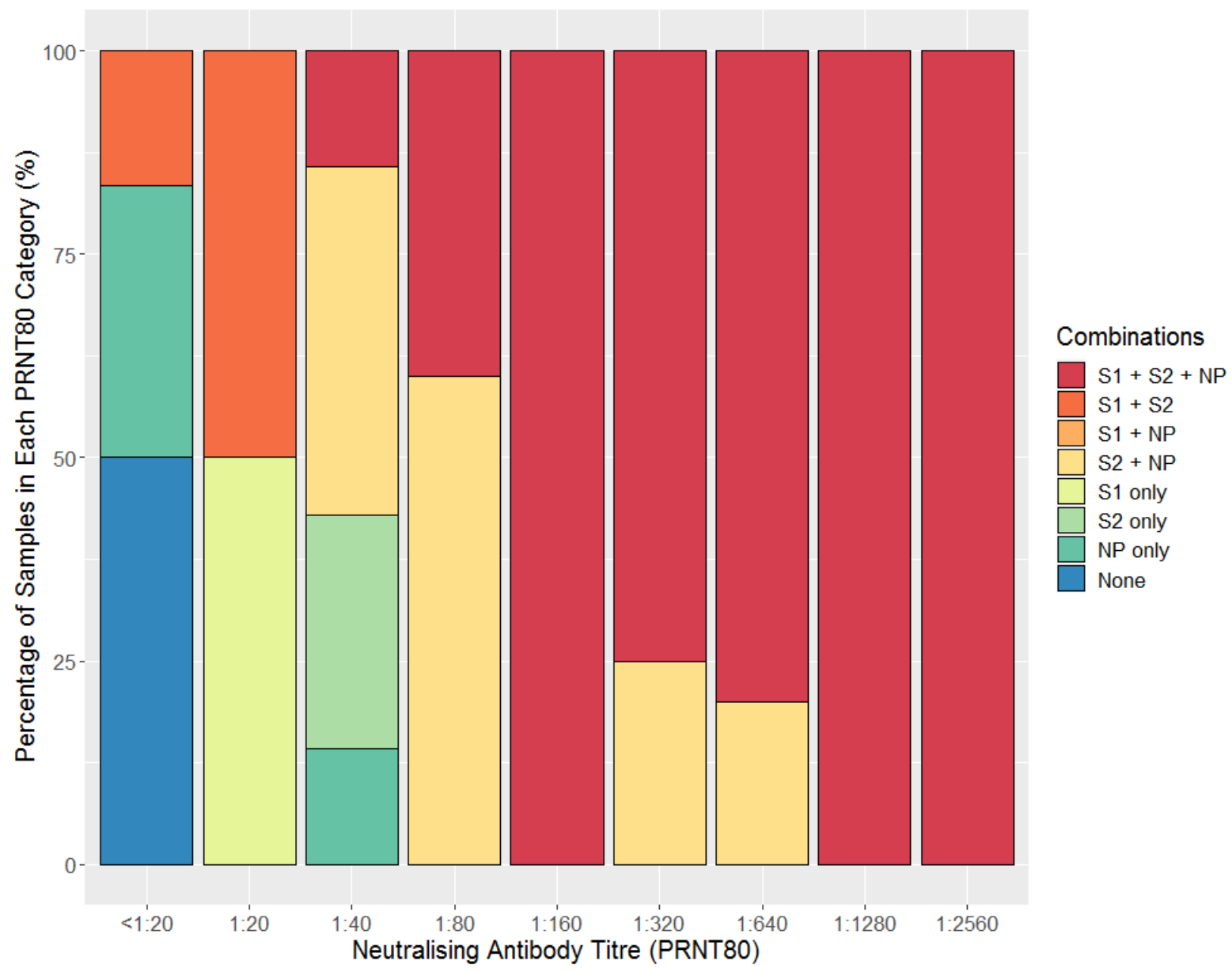

376 Figure 3: Percentage of samples in PRNT 80 categories binding to different antigen combinations by

377 Western Blot. S1 = spike protein subunit 1; S2 = spike protein subunit 2; NP = nucleocapsid protein 
Figures

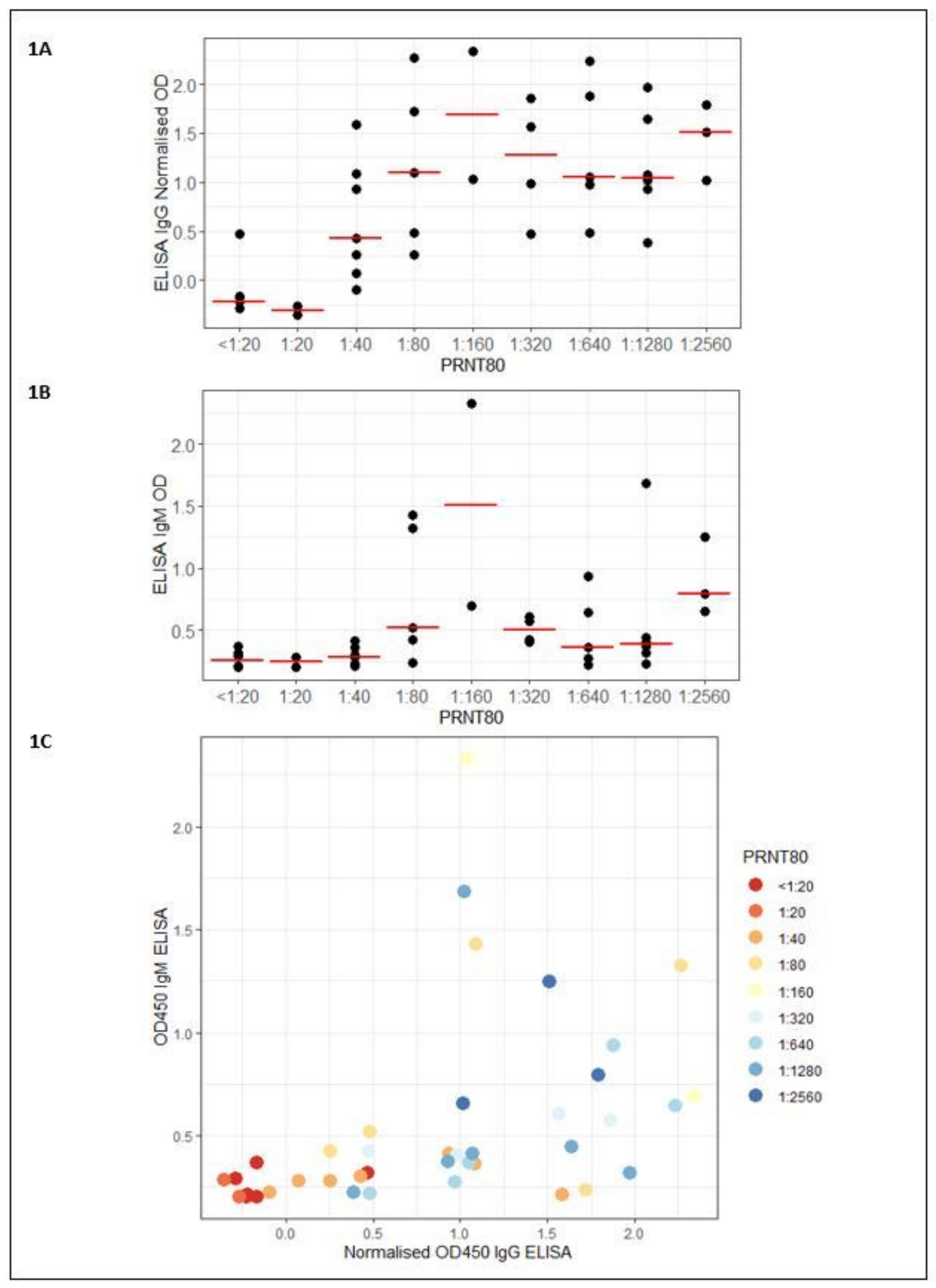

Figure 1

IgG NOD450 (1A) and IgM OD450 (1B) by PRNT80 category, and paired IgG and IgM ELISA results for each serum sample (1C) 

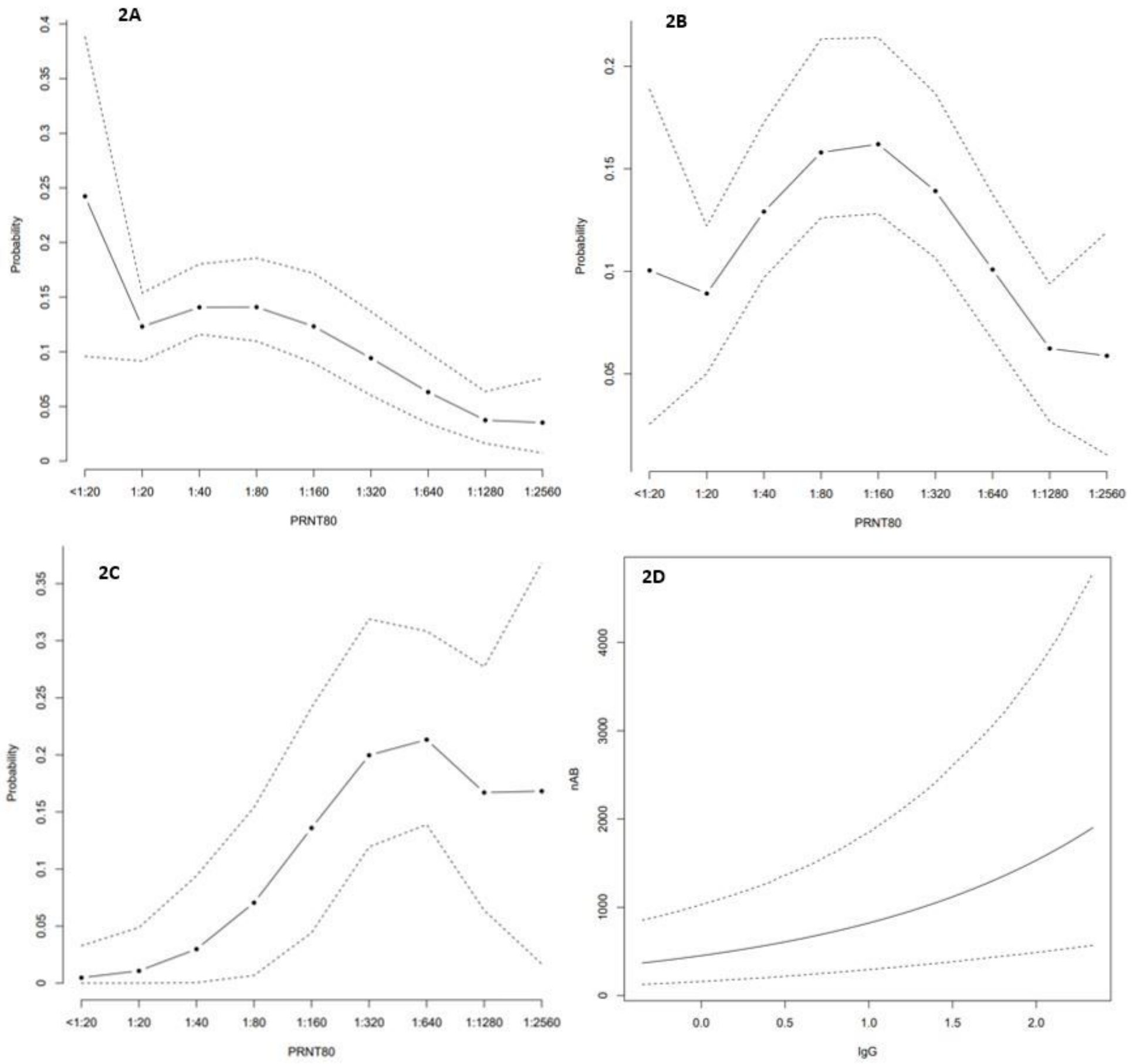

Figure 2

PRNT80 probability estimates by IgG ELISA result 


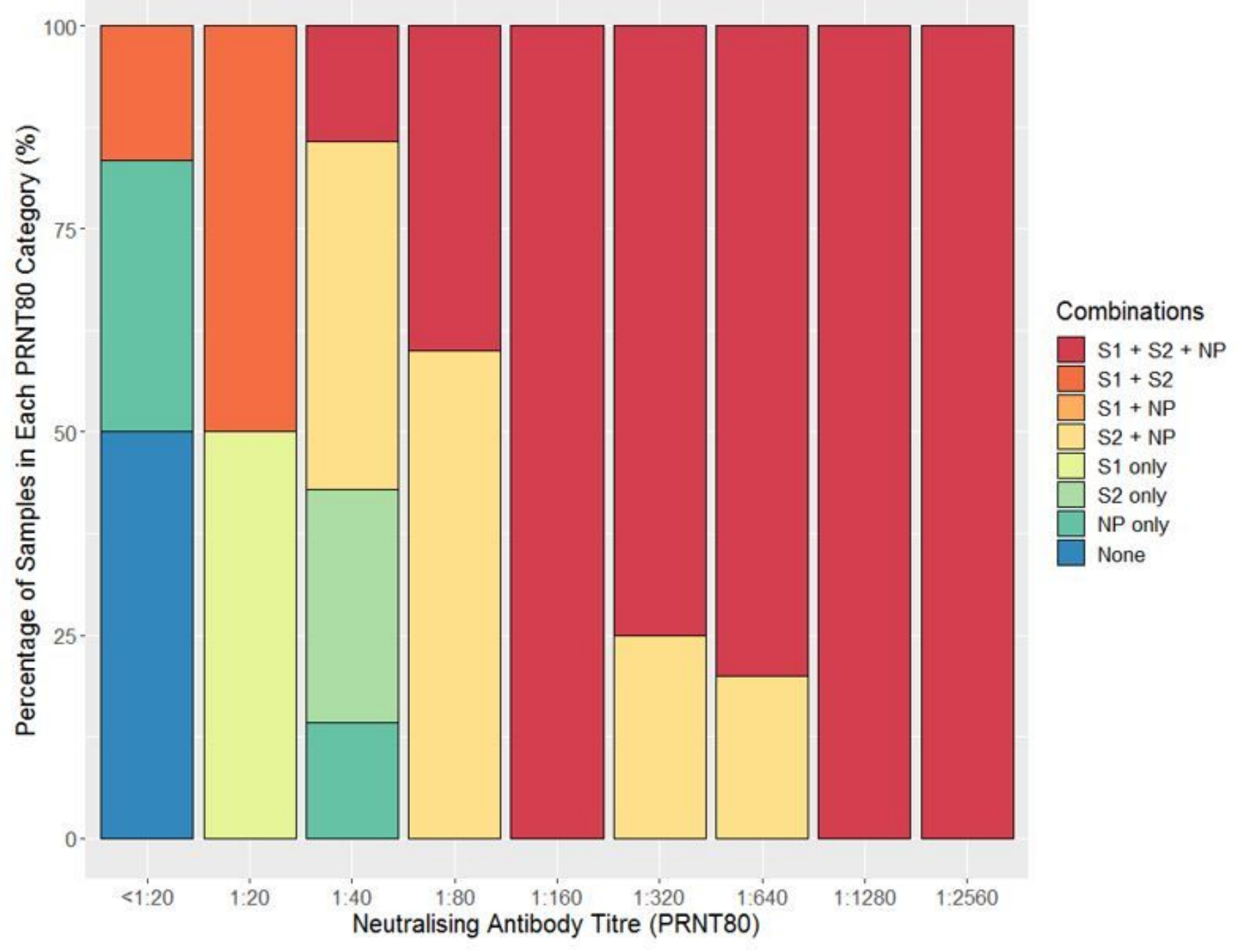

Figure 3

Percentage of samples in PRNT80 categories binding to different antigen combinations by Western Blot

\section{Supplementary Files}

This is a list of supplementary files associated with this preprint. Click to download.

- Supplementary1.pdf 